FORSCHUNGSZENTRUM

ROSSENDORF e.v.

FZR

\title{
FZR-162
}

January 1997

Preprint

\author{
T. Gorin, F.-M. Dittes, M. Müller, \\ I. Rotter and T. H. Seligman
}

\section{Correlations between resonances in \\ a statistical scattering model}




\title{
Correlations between resonances in a statistical scattering model
}

\author{
T. Gorin, ${ }^{1,2}$ F.-M. Dittes, ${ }^{2,3}$ M. Müller, ${ }^{4}$ I. Rotter, ${ }^{1,2}$ and T. H. Seligman ${ }^{4}$ \\ ${ }^{1}$ Technische Universität Dresden, Institut für Theoretische Physik, D-01062 Dresden, Germany \\ ${ }^{2}$ Forschungszentrum Rossendorf, Institut für Kern- und Hadronenphysik, D-01314 Dresden, Germany \\ ${ }^{3}$ Max-Planck-Institut für Physik Komplexer Systeme, D-01187 Dresden, Germany \\ ${ }^{4}$ Centro Internacional de Ciencias, Cuernavaca, Mexico
}

(Received 21 February 1997)

\begin{abstract}
The distortion of the regular motion in a quantum system by its coupling to the continuum of decay channels is investigated. The regular motion is described by means of a Poissonian ensemble. We focus on the case of only a few channels $K<10$. The coupling to the continuum induces two main effects, due to which the distorted system differs from a chaotic system (described by a Gaussian ensemble): (i) The width distribution for large coupling becomes broader than the corresponding $\chi_{K}^{2}$ distribution in the Gaussian orthogonal ensemble case. (ii) Due to the coupling to the continuum, correlations are induced not only between the positions of the resonances but also between positions and widths. These correlations remain even in the strong coupling limit. In order to explain these results, we relate the width of a trapped resonance state to the distance between its neighboring levels and derive an asymptotic expression for the width distribution for the one channel case. [S1063-651X(97)08308-6]
\end{abstract}

PACS number(s): 05.30.Ch, 05.40.+j, 03.65.Nk

\section{INTRODUCTION}

Resonances are states of an open quantum mechanical system, in which the internal dynamics of the underlying closed system is disturbed by the coupling to the decay channels. For the small coupling strength the widths of the resonances increase with growing coupling, while their positions remain almost unchanged [1-3]. Therefore the degree of overlap of neighboring resonances increases. This gives rise to interferences and the internal dynamics may suffer dramatic changes. If the coupling strength passes over a critical value, a segregation of the decay widths occurs. Finally, for large couplings, $K$ rapidly decaying modes and $N-K$ narrow resonances result from this interference process [4-6]. In the following we stick to the statistical approach $[7,8]$, which is more appropriate for the case of many resonances. Then the internal dynamics of the system in consideration is modeled by a matrix ensemble. This may be chosen to describe regular and chaotic motion, respectively.

The distortion of regular motion by coupling a closed quantum system to the continuum has up to now received little attention theoretically [9-11] and experimentally [1214]. In contrast to that, such problems draw considerable attention in classical and semiclassical theories (e.g., [15] and references therein). It would be desirable to connect the approaches from both fields. The present work is meant to be a first step along that way.

In [10], the regular motion is described by a Poissonian orthogonal ensemble (POE). Its perturbation due to its coupling to the continuum of decay channels is investigated as a function of a parameter $\alpha$, which characterizes the coupling strength. Level repulsion appears at large $\alpha$ where the widths have already segregated. There, with an increasing number of channels, the correlations in the positions of the resonances approach those, characteristic for the Gaussian orthogonal ensemble (GOE). At the same time the distribution of widths experiences a considerable broadening in compari- son to the $\chi_{K}^{2}$ distributed widths in the GOE case. This broadening is quasi-independent of the number of channels. A similar effect has been found in the transition-strength distribution of a closed system $[16,17]$. There the broadening (or increase in entropy) is explained by the onset of chaos. Note that a broad width distribution implies deviations from the exponential decay law $[18,19]$.

A physical example of a regular system, coupled to the continuum, is investigated in $[11,13]$ on experimental and theoretical grounds. The regular motion of the microwaves inside a rectangular resonator is disturbed by an attached antenna, which defines the decay channel. Theory predicts the effective coupling of the antenna to the resonator to increase with the frequency. The experimental data was obtained in the regime of strong external coupling, where the resonance widths have already segregated. It should be possible to apply the results obtained in the present paper to this experimental setup.

In the following we compare the results for a regular system described by a POE with those of a chaotic system described by a GOE, both coupled in the same way to the continuum. Our interest focuses on the width distribution and the correlations induced by the coupling of the system to the continuum of decay channels.

In Sec. II, we describe the statistical model used in our investigations, as well as some technicalities, concerning the numerical studies performed. A redistribution of the spectroscopic properties takes place in the system if its coupling to the continuum of decay channels is sufficiently strong. We sketch this mechanism (trapping effect) and give the formula for the mean width of the long-lived resonances in Sec. III. The distribution of the widths most characteristic of the trapping effect is considered in Sec. IV. In Sec. V, we present our results for the correlations in the positions of the resonances while correlations between widths and positions are discussed in Sec. VI. Some conclusions are drawn in Sec. VII. 


\section{MODEL}

\section{A. Projection formalism}

Our analysis proceeds from the statistical model [3,5]. By using the projection operator technique [3] we couple $N$ bound states $\left(\left|\Phi_{i}\right\rangle, i=1, \ldots, N\right)$ to $K$ common decay channels $\left(\left|\chi_{c}(E)\right\rangle, c=1, \ldots, K\right)$ via the two body residual interaction $V$. We are mainly interested in the bound state part of the system and its perturbations by the environment of decay channels. To this end, we investigate the statistical properties of the system as a function of the coupling strength to the channels.

The total Hamiltonian of the whole quantum mechanical system consists of three different parts: the Hamiltonian $H$ describing the closed system of bound states, a term describing the $K$ continua, and a third term which specifies the coupling between the two subspaces:

$$
\begin{aligned}
H_{\mathrm{tot}}= & \sum_{i, j=1}^{N}\left|\Phi_{i}\right\rangle H_{i j}\left\langle\Phi_{j}\left|+\sum_{c=1}^{K} \int d E\right| \chi_{c}(E)\right\rangle E\left\langle\chi_{c}(E)\right| \\
& +\sum_{c=1}^{K} \sum_{i=1}^{N} \int d E\left[\left|\Phi_{i}\right\rangle \mathcal{V}_{i}^{c}(E)\left\langle\chi_{c}(E)\right|+\text { H.c. }\right] .
\end{aligned}
$$

Here the $\mathcal{V}_{i}^{c}(E)$ are the coupling matrix elements between the bound states $\left|\Phi_{i}\right\rangle$ and the scattering states $\left|\chi_{c}(E)\right\rangle$. They can be understood as the components of an $N$-dimensional vector $\mathcal{V}^{c}$. Its norm is a measure of the coupling strength to the channel $c$. Direct reactions are neglected. We consider scattering systems with time reversal invariance, so we can restrict ourselves to real matrices $H_{i j}$ and $\mathcal{V}_{i}^{c}(E)$.

As shown in [3] the $S$ matrix of such a system reads

$$
S_{a b}(E)=\delta_{a b}-2 \pi i \sum_{i, j=1}^{N} \mathcal{V}_{i}^{a}(E) \mathcal{G}_{i j}(E) \mathcal{V}_{j}^{b}(E) \text {, }
$$

where $\mathcal{G}$ is the propagator in the perturbed system $H+W^{\text {ext }}$,

$$
\mathcal{G}=\left[E-H-W^{\mathrm{ext}}\right]^{-1},
$$

and the generally complex operator $W^{\text {ext }}$ describes the perturbations from outside,

$$
W_{i j}^{\mathrm{ext}}=\sum_{c=1}^{K} \int d E^{\prime} \frac{\mathcal{V}_{i}^{c}\left(E^{\prime}\right) \mathcal{V}_{j}^{c}\left(E^{\prime}\right)}{E+i \varepsilon-E^{\prime}} .
$$

According to Eq. (2), the pole structure of the $S$ matrix is given by the eigenvalues of the operator $H+W^{\text {ext }}$. We suppose, that the quantities $\mathcal{V}_{i}^{c}\left(E^{\prime}\right)$ are (almost) independent of the excitation energy, which is justified if one considers a finite energy region sufficiently far from the reaction thresholds. With this assumption Eq. (4) simplifies

$$
W_{i j}^{\mathrm{ext}}=-\frac{i}{2} \eta \sum_{c=1}^{K} V_{i c} V_{j c},
$$

where we replaced $\mathcal{V}_{i}^{c}$ by $\sqrt{2 \pi / \eta} V_{i c}, V_{i c}$ being a $N \times K$ matrix with on the average unit column vectors, and $\eta$ being the total coupling strength per channel. Then the $S$ matrix takes the form

$$
S(E)=\mathbf{1}-i \eta V^{\dagger}\left[E-H_{\mathrm{eff}}\right]^{-1} V .
$$

Its complex poles are given by the eigenvalues $\widetilde{\mathcal{E}}_{n}$ of the non-Hermitian Hamiltonian

$$
H_{\mathrm{eff}}=H-\frac{i}{2} \eta V V^{\dagger}
$$

with

$$
\widetilde{\mathcal{E}}_{n}=E_{n}-\frac{i}{2} \Gamma_{n}
$$

Under the influence of the external coupling, the eigenstates of the Hermitian matrix $H$ are turning into resonances with a finite lifetime. In the case of nonoverlapping resonances, the real part $E_{n}$ and the imaginary part $\Gamma_{n}$ in Eq. (8), give the position and the total decay width of the resonance. We will stick to these terms in all cases, remembering the definition as poles of the $S$ matrix.

\section{B. Scattering ensemble}

Following [5] we represent $H_{\text {eff }}$ in the eigenbasis of its Hermitian part $H$. Demanding orthogonal invariance of the scattering ensemble, the matrix ensemble is characterized solely by the eigenvalue distribution of $H$, and the antiHermitian part $-i \eta / 2 \quad V V^{\dagger}$ is independent on the specific choice of the ensemble. In all cases $V$ consists of $K$ random column vectors. For large $N$ this implies the elements of $V$ being independent random Gaussian variables with

$$
\left\langle V_{i a}\right\rangle=0, \quad\left\langle V_{i a}^{2}\right\rangle=\frac{1}{N}
$$

It follows that the matrix elements of the anti-Hermitian part $\left\langle\left(V V^{\dagger}\right)_{i j}^{2}\right\rangle$ are of the order $N^{-2}$.

The pole distribution for the $H_{\text {eff }}$ ensemble may be expressed by [5]

$$
\begin{aligned}
P\left(\left\{E_{j}, \Gamma_{j}\right\}\right)= & C \int d^{N}\left\{\varepsilon_{k}\right\} d^{N K}\left\{V_{l a}\right\} \\
& \times \prod_{j} \delta\left(\operatorname{det}\left[H_{\mathrm{eff}}-E_{j}+\frac{i}{2} \Gamma_{j}\right]\right) \prod_{m<n}\left|\varepsilon_{m}-\varepsilon_{n}\right|^{\beta} \\
& \times \exp \left(-\frac{N}{a^{2}} \sum_{n} \varepsilon_{n}^{2}\right) \exp \left(-\frac{N}{2} \sum_{n, a} V_{n a}^{2}\right) .
\end{aligned}
$$

$C$ is a normalization constant. The indices $j, k, l, m$, and $n$ are running from $1, \ldots, N$, while $a$ numbers the channels $1, \ldots, K$. The integration runs over the whole ensemble parametrized by the eigenvalues $\left\{\varepsilon_{k}\right\}$ of $H$ and the coefficients $\left\{V_{l a}\right\}$ of the coupling matrix. By means of the $\delta$ functions, the positions and widths of the resonances are introduced as the new variables of the distribution. The first part in the last line represents the eigenvalue distribution of $H$, where $a$ is related to the range of the spectrum. The last part in this line represents the distribution of the matrix elements of $V$. 
The parameter $\beta>-1$ controls the degree of level repulsion. $\beta=1$ refers to the GOE and $\beta=0$ to the POE case. $\beta \mapsto \infty$ describes a completely rigid spectrum (harmonic oscillator), while $\beta<0$ refers to a spectrum showing level clustering. Note that the cases $\beta=2$ and $\beta=4$ do not describe the unitary and symplectic ensembles, because we always work with real matrices $V$, demanding orthogonal invariance.

In the following we restrict ourselves to the cases $\beta=1$ (GOE) and $\beta=0$ (POE). For the GOE the level density is described by Wigner's semicircle [21], $a$ being its radius. For the POE Eq. (10) would produce a Gaussian shaped level density. But in order to compare the correlations in both ensembles we find it more convenient to have equal level densities. Therefore, we choose the $\left\{\varepsilon_{j}\right\}$ in the POE case as distributed according to Wigner's semicircle law, too.

Unless stated otherwise, all numerical calculations were done by diagonalizing $H_{\text {eff }}$ of dimension $N=300$ and with $K=3$ channels being approximately orthogonal to one another, due to the centralized distribution of their components. $V$ is a $N \times K$ matrix with random Gaussian coefficients with zero mean and variance $1 / N$. The calculations are performed for 50 matrices chosen from the GOE or the POE, respectively. Then the statistical observables are calculated as averages over the central part of the spectrum and over the ensemble (the 50 matrices) simultaneously. In the case of correlation measures $\left(\Sigma^{2}\right.$ and $\Sigma_{\mathrm{g}}^{2}$ in Sec. V and Sec. VI) the positions of the resonances are unfolded to the equal mean level spacing $d=1$.

\section{RESONANCE TRAPPING}

\section{A. Separation of time scales}

One of the specific properties of open quantum systems is the formation of separate time scales. In the strong coupling regime $\eta \gg 1, K$ resonances become very broad by "trapping" the remaining $N-K$ ones [5,22]. Mathematically the trapping effect is caused by the fact that the rank of the Hermitian part of $H_{\text {eff }}$ is $N$ while that of the anti-Hermitian part $-(i \eta / 2) V V^{\dagger}$ cannot be larger than $K<N$ due to its dyadic form (7). Thus we have the following picture: At weak coupling the resonances do not overlap: $\langle\Gamma\rangle \ll D$, where $D$ is the mean level distance, and the anti-Hermitian part of $H_{\text {eff }}$ may be treated by the first order perturbation theory. Then the widths of the resonances are well approximated by the diagonal elements of $V V^{\dagger}$ and have comparable magnitude,

$$
\Gamma_{j}=\eta \sum_{a} V_{i a}^{2}
$$

In the opposite case of strong coupling, the resonances overlap and the anti-Hermitian part $-(i \eta / 2) V V^{\dagger}$ dominates the behavior of the decaying system. The appropriate basis is now the eigenbasis of $V V^{\dagger}$ consisting of $K$ channel vectors (the columns of $V$ ) and $N-K$ vectors, spanning the kernel of $V V^{\dagger}$. The channel vectors have the common eigenvalue $\eta$. The widths of the remaining states are zero.

In order to get some information about the magnitude of the widths of the trapped resonances it is necessary to go beyond the simple ansatz sketched above. This is done in the following subsection.

\section{B. Mean width of the trapped resonances}

We will calculate the mean width of the trapped resonances in the strong coupling regime following [5,23]. In addition to what has been done there, we explicitly show the applicability to the POE case [in fact it may be applied to any orthogonal invariant ensemble for arbitrary $\beta$ as defined in Eq. (10)]. In order to exploit the orthogonal invariance we need the first and second moments of the distributions of the matrix elements in a generic (random) basis. For the GOE they are [21]

$$
\left\langle H_{i j}\right\rangle=0, \quad\left\langle H_{i j}^{2}\right\rangle=\left\{\begin{array}{cc}
\frac{a^{2}}{2 N}, & i=j \\
\frac{a^{2}}{4 N}, & i \neq j .
\end{array}\right.
$$

For the POE we calculate these moments starting from the level distribution, and applying a random orthogonal transformation $O$

$$
H=O \operatorname{diag}\left\{\varepsilon_{j}\right\} O^{T}, \quad\left\langle O_{i j}\right\rangle=0, \quad\left\langle O_{i j}^{2}\right\rangle=\frac{1}{N}
$$

So every element of $H$ is defined by

$$
H_{i j}=\sum_{k} O_{i k} \varepsilon_{k} O_{k j}
$$

Due to the centralized distribution of the $\left\{\varepsilon_{i}\right\}$ the first moment $\left\langle H_{i j}\right\rangle$ is zero again. Then we calculate the average of the squares $H_{i j}^{2}$. As the eigenvalues are not correlated, we get

$$
\begin{aligned}
& \left\langle H_{i j}^{2}\right\rangle \\
& =\left\{\begin{array}{l}
\left\langle\sum_{k} O_{i k}^{4} \varepsilon_{k}^{2}+\sum_{k \neq l} O_{i k}^{2} O_{i l}^{2} \varepsilon_{k} \varepsilon_{l}\right\rangle=\frac{3}{N}\left\langle\varepsilon^{2}\right\rangle=\frac{3 a^{2}}{4 N}, \quad i=j \\
\left\langle\sum_{k} O_{i k}^{2} \varepsilon_{k}^{2} O_{j k}^{2}\right\rangle=\frac{1}{N}\left\langle\varepsilon^{2}\right\rangle=\frac{a^{2}}{4 N} \quad i \neq j
\end{array}\right.
\end{aligned}
$$

Note that only the diagonal elements differ from the GOE case in their second moment.

In the following we show that the mean width of the trapped resonances can be calculated from the second moments of the matrix elements $H_{i j}$. To this end we turn to the channel representation of $H_{\text {eff }}$. In this basis the channel vectors are the first $K$ canonical vectors and the remaining $N-K$ vectors constitute a basis for the kernel of $V V^{\dagger}$. Then

$$
H_{\mathrm{eff}}=H-\frac{i}{2} \eta\left(\begin{array}{cc}
\delta_{a b} & 0 \\
0 & 0
\end{array}\right)
$$


$a$ and $b$ again serve as channel indices, while the greek letters $\mu$ and $\nu$ are used in connection with the long-lived resonances. We diagonalize $H$ in the kernel of $V V^{\dagger}$ by the following orthogonal transformation:

$$
P=\left(\begin{array}{cc}
\delta_{a b} & 0 \\
0 & P_{\mu \nu}
\end{array}\right), \quad\left(\begin{array}{cccc}
H_{a b}-\frac{i \eta}{2} \delta_{a b} & & * & \\
& \varepsilon_{K+1}^{\prime} & & 0 \\
* & & \ddots & \\
& 0 & & \varepsilon_{N^{\prime}}
\end{array}\right) .
$$

Note that $\left\{\varepsilon_{\nu}^{\prime}\right\}$ differ from the eigenvalues $\left\{\varepsilon_{j}\right\}$ of $H . D$ is symmetric and therefore the submatrices denoted by * are transposed to each other,

$$
D_{\mu b}=\sum_{k, l=1}^{N} P_{k \mu} H_{k l} P_{l b}=\sum_{\nu=K+1}^{N} P_{\nu \mu} H_{\nu b} .
$$

The $P_{\nu \mu}$ are only correlated with the matrix elements $H_{\mu \nu}$ as they are diagonalizing this submatrix, but not with $H_{\mu b}$. Therefore one may average over these quantities independently.

Now we calculate approximate eigenvalues of $D$ by applying a Jacobi transformation of dimension $K+1$ [24]. Such a transformation is trace invariant (this is important because the total width should be conserved). So we look for the zero of the following determinant (for arbitrary $K+1 \leqslant \mu \leqslant N$ ):

$$
\operatorname{det}\left(\begin{array}{cc}
\widetilde{\mathcal{E}}_{\mu}-\varepsilon_{\mu} & D_{\mu 1}, \ldots, D_{\mu K} \\
D_{\mu 1} & \\
\vdots & \widetilde{\mathcal{E}}_{\mu}-H_{a b}+\frac{i \eta}{2} \delta_{a b} \\
D_{\mu K} &
\end{array}\right)=0 .
$$

Taking into account only the highest orders in $\eta$, we arrive at

$$
\widetilde{\mathcal{E}}_{\mu}-\varepsilon_{\mu}+\sum_{a} \frac{D_{\mu a}^{2}}{\mathcal{E}-H_{a a}+\frac{i \eta}{2}}+\odot\left(\eta^{-2}\right)=0
$$

We can read the approximate position and width of a trapped resonance from the real and the imaginary part of $\widetilde{\mathcal{E}}_{\mu}$. For $\eta \mapsto \infty$ we get

$$
E_{\mu}=\varepsilon_{\mu}^{\prime}, \quad \Gamma_{\mu}=\frac{4}{\eta} \sum_{a} D_{\mu a}^{2}
$$

The final step is the calculation of the ensemble mean of $D_{\mu a}^{2}$ [using Eqs. (12) and (15)]

$$
\left\langle D_{\mu a}^{2}\right\rangle=\left\langle\sum_{\nu} P_{\nu \mu}^{2} H_{\nu a}^{2}\right\rangle=\left\langle H_{\nu a}^{2}\right\rangle=\frac{\left\langle\varepsilon^{2}\right\rangle}{N} .
$$

From that it follows that the mean width for the trapped resonances is

$$
\left\langle\Gamma_{\mu}\right\rangle=\frac{4}{\eta}\left\langle\sum_{a} D_{\mu a}^{2}\right\rangle=\frac{4 K}{N \eta}\left\langle\varepsilon^{2}\right\rangle=\frac{K a^{2}}{N \eta} .
$$

Note that the diagonal elements of $H$ do not enter into the calculation of $\left\langle\Gamma_{\mu}\right\rangle$, so that Eq. (23) holds for both the GOE and the POE.

Although the matrix elements of the Hermitian and the anti-Hermitian part scale differently with $N$ [compare Eqs. (9) and (12)] the norm (i.e., the distance between the smallest and the largest eigenvalue) of both matrices is of the same order in $N$. Therefore an appropriate parameter to indicate the relative strength of the continuum coupling is the ratio of the two norms. Equivalently it can be defined as the average degree of overlapping, given by the ratio of the summed width per channel and the "width" of the spectrum,

$$
\kappa=\frac{\eta}{2 \sqrt{\left\langle\varepsilon^{2}\right\rangle}}
$$

For the trapped resonances this overlapping parameter is

$$
\kappa_{f}=\frac{(N-K)\left\langle\Gamma_{\mu}\right\rangle}{2 K \sqrt{\left\langle\varepsilon^{2}\right\rangle}} .
$$

Then it follows from Eq. (23) that in the limit $N \mapsto \infty$

$$
\kappa_{f}=\frac{N-K}{N} \frac{2 \sqrt{\left\langle\varepsilon^{2}\right\rangle}}{\eta} \mapsto \kappa^{-1} .
$$

Assuming ergodicity [as expressed by the randomness of the eigenbasis (13)] the ensemble average may be replaced by the spectral average. Then Eq. (23) may even be applied to a single system. In this case it may serve as a test for the trapping scenario to occur in the system considered: Eq. (23) relates the product of the average width $\left\langle\Gamma_{\mu}\right\rangle$ of the trapped resonances and the average width $\eta$ of the broad resonances to the variance of the level density $\left\langle\varepsilon^{2}\right\rangle$.

For small $\kappa$ one can still consider the mean width of the $N-K$ smallest resonances in order to have $\kappa_{f}$ well defined globally. Then this quantity will be equal to the mean width of all resonances, if $\kappa$ is sufficiently small. Therefore

$$
\kappa_{f}= \begin{cases}\kappa, & \kappa \ll 1 \\ \kappa^{-1}, & \kappa \gg 1 .\end{cases}
$$

We define the weak coupling regime by $\kappa \ll 1$, so that the upper part of Eq. (27) is fulfilled, and the strong coupling regime by $\kappa \gg 1$ so that the lower part becomes valid. In between (the critical region) the redistribution takes place.

For illustration, in Fig. 1 the trapping process is portrayed for both the GOE and the POE, from the low coupling to the high coupling regime. We plot $\kappa_{f}$ versus $\kappa$ as defined in Eq. (24). A double-log plot is used in order to demonstrate the proportionality relation (27). As the level density is the same for the GOE and the POE (cf. Sec. II B), the data points of both the GOE and the POE case follow the same line for all coupling strengths within the numerical accuracy. The asymmetry on the left side of the plot is due to the finite dimension $N=300$ in our calculations. 


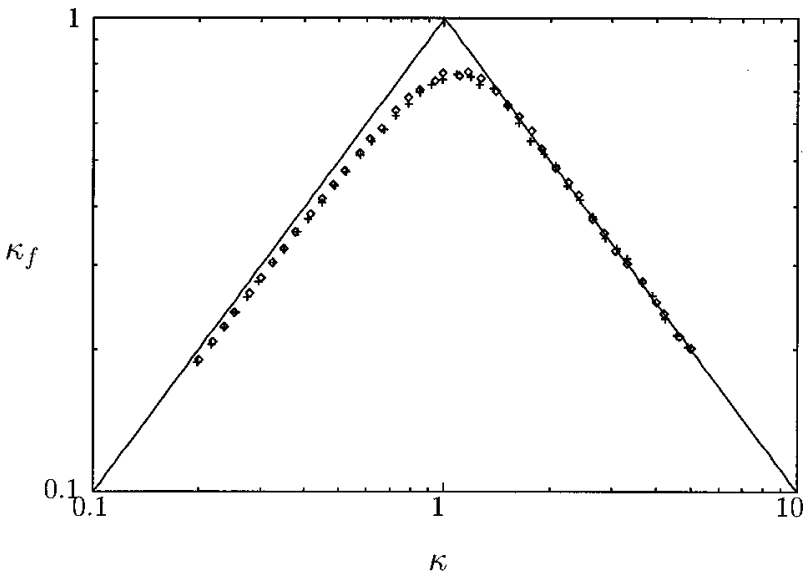

FIG. 1. The overlapping parameter $\kappa_{f}$ of the trapped resonances vs the overlapping parameter $\kappa$ of all resonances. The diamonds denote data points for the GOE, the crosses those for the POE. The solid lines show the two asymptotics $\kappa$ and $\kappa^{-1}$.

The redistribution between the two scenarios at small and large coupling $\eta$ occurs rather promptly at $\kappa \approx 1$. Here the $K$ broad poles appear, which will almost share the total sum of the widths, whereas the remaining $N-K$ resonances will become more and more long lived in the strong coupling regime. The point $\kappa=1$, at which $\kappa_{f}$ reaches its maximal value, is called the critical point. Note the peculiar properties of the transmission coefficient $[25,9]$ and of the width distribution [20] at this point.

\section{WIDTH DISTRIBUTION}

In the weak coupling regime the widths are approximately given by the diagonal elements of the coupling matrix $V V^{\dagger}$ (11). These are sums of $K$ random Gaussian variables and, therefore, $\chi_{K}^{2}$ distributed when normalized to unit mean

$$
p(y)=\chi_{K}^{2}(y)=\frac{(K / 2)^{K / 2}}{\Gamma(K / 2)} y^{K / 2-1} e^{-K / 2 y} .
$$

For the GOE the same distribution holds in the strong coupling region as shown for the one channel case in [5], by calculating the joint probability distribution for the complex eigenvalues of $H_{\text {eff }}$ explicitely. For the POE the width distribution becomes much broader.

The numerical results are shown in Fig. 2. Here a series of width distributions for the GOE (left side) and the POE (right side) for increasing coupling strength $\kappa$ (from top to bottom) is given. When $\kappa>1$, only the long-lived resonances were taken into account. In each diagram, we plotted the numerical data (histogram), and the $\chi_{K=3}^{2}$ distribution (dashed line). For the POE at $\kappa=100$ a best fit $\chi_{q}^{2}$ distribution (dotted line) is given. This distribution is calculated by replacing in Eq. (28) $K$ by a real parameter $q$ and performing a $\chi^{2}$ fit in order to find the best estimate for $q$.

For both ensembles the GOE and the POE, the width distributions undergo strong deformations in the critical region, but become stationary again in the strong coupling limit. In contrast to the GOE case, which returns to its old

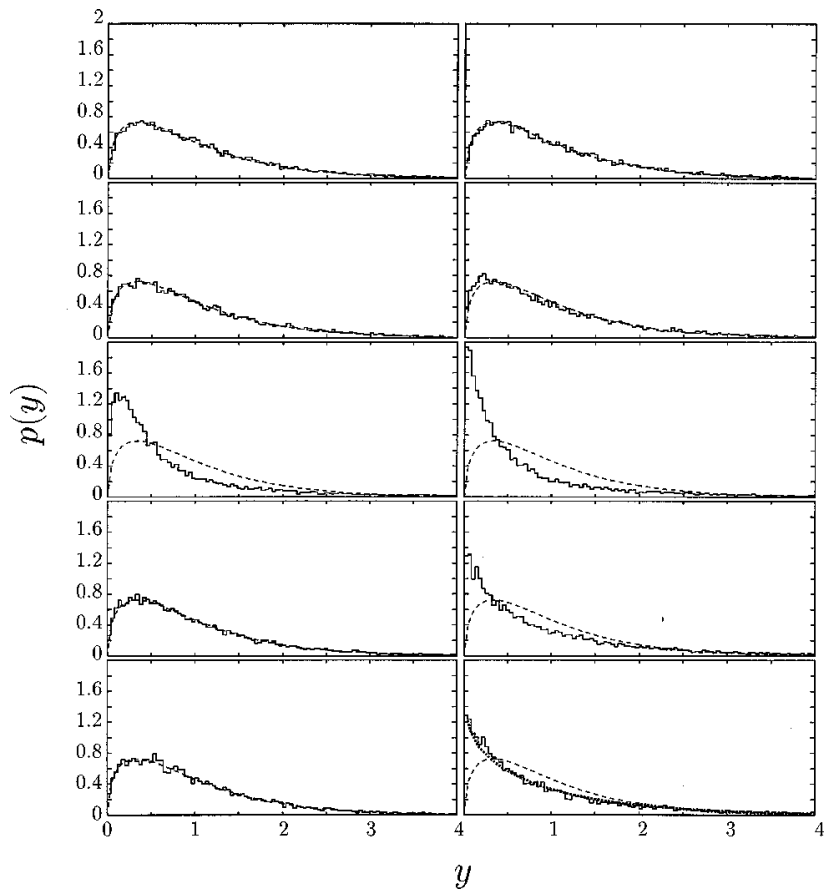

FIG. 2. Normalized width distributions for the GOE (left side) and the POE (right side) for different coupling strengths $\kappa=0.01,0.1,1,10$, and 100 (from top to bottom). Histogram of the width distribution obtained numerically (solid line). $\chi_{K=3}^{2}$ distribution (dashed line). Best fit $\chi_{q=1.7}^{2}$ distribution (dotted line) on the bottom left.

shape, the distribution for the POE remains much broader. It is in good agreement with the best fit $\chi_{q}^{2}$ distribution with $q=1.7$.

Further investigations of the POE at strong coupling for more channels led to the following interesting behavior (cf. Fig. 3): The coupling to continuum leads to a broadening of the width distribution. For increasing number of channels $K$ its variance approaches double what it was at small coupling (or double what it is in the corresponding GOE case).

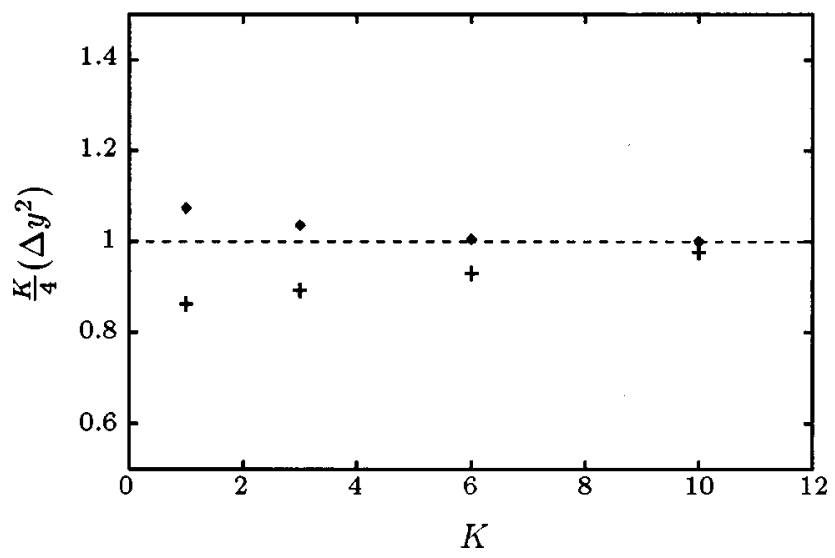

FIG. 3. Relative deviations of the variance of the width distributions (POE, strong coupling) for $K=1,3,6$, and 10 channels. The diamonds denote the variances calculated directly from the data, whereas the crosses denote $2 / q$, the variances of the best fit $\chi_{q}^{2}$ distributions. These values were divided by $4 / K$, which seems to be the limit value for the variance at $K \mapsto \infty$. 
Whereas the variances of the best fit distributions systematically underestimate the factor 2 , the numerical variances overestimate it.

In order to understand the broadening of the width distributions due to the coupling to continuum, we consider the one channel case in more detail. The $S$ matrix (6), with complex argument $\mathcal{E}$, can be equivalently written as [23]

$$
S(\mathcal{E})=\frac{1-i R}{1+i R}, \quad R=\frac{\eta}{2} V^{+} \frac{1}{\mathcal{E}-H} V .
$$

From that it follows that the poles of the $S$ matrix are given by the zeros of the function

$$
f(\mathcal{E})=1+\frac{i}{2} \eta \sum_{i} \frac{v_{i}^{2}}{\mathcal{E}-\varepsilon_{i}} .
$$

The $v_{i}$ are the Gaussian random coefficients of the coupling matrix (11) with variance $1 / N$ and the $\varepsilon_{i}$ are the real eigenvalues of $H$. We may consider the real and the imaginary part of $f$ separately. As we are interested in the case $\eta \mapsto \infty$ we only keep the highest order of $\eta$, making use of $\Gamma \sim \eta^{-1}$ (23). Using the notation $\mathcal{E}=E-(i / 2) \Gamma$ for the poles one arrives at $[11]$

$$
\sum_{i} \frac{v_{i}^{2}}{E-\varepsilon_{i}}=0, \quad \frac{4}{\eta \Gamma}=\sum_{i} \frac{v_{i}^{2}}{\left(E-\varepsilon_{i}\right)^{2}} .
$$

Between every two neighboring levels there has to settle down one pole with increasing $\eta$ due to the structure of Eq. (31). Choosing an arbitrary one of them and taking into account only its two neighboring levels, we find the following formula for its width (a detailed derivation is given in Appendix A):

$$
\Gamma=\frac{d_{0}^{2} s^{2}}{\eta\left(v_{1}^{2}+v_{2}^{2}\right)}\left(1-\tau^{2}\right), \quad \tau=\frac{v_{2}^{2}-v_{1}^{2}}{v_{1}^{2}+v_{2}^{2}} .
$$

Here $s$ is the distance between the two consecutive levels measured in units of the mean level distance $d_{0}$ in the center of the spectrum. In our case of a semicircular level density with radius $a, d_{0}=\pi a /(2 N)$. In Appendix B we calculate the distribution of the trapped widths normalized by their mean $\left\langle\Gamma_{\nu}\right\rangle$ (23) for the GOE and the POE case. This is done by evaluating the following integral:

$$
\begin{aligned}
p(y= & \left.\Gamma /\left\langle\Gamma_{\nu}\right\rangle\right)=\frac{N}{2 \pi} \int d s P(s) \int d v_{1} d v_{2} e^{-N / 2\left(v_{1}^{2}+v_{2}^{2}\right)} \\
& \times \delta\left[y-\frac{\pi^{2} s^{2}}{4 N} \frac{1-\tau^{2}}{v_{1}^{2}+v_{2}^{2}}\right] .
\end{aligned}
$$

Here $P(s)$ is the nearest neighbor distribution for the ensemble in consideration. The result for the GOE is

$$
p_{\mathrm{G}}(y)=\frac{1}{\sqrt{2 \pi y}}\left(1+\frac{2 y}{\pi}\right)^{-3 / 2},
$$

and for the POE it is

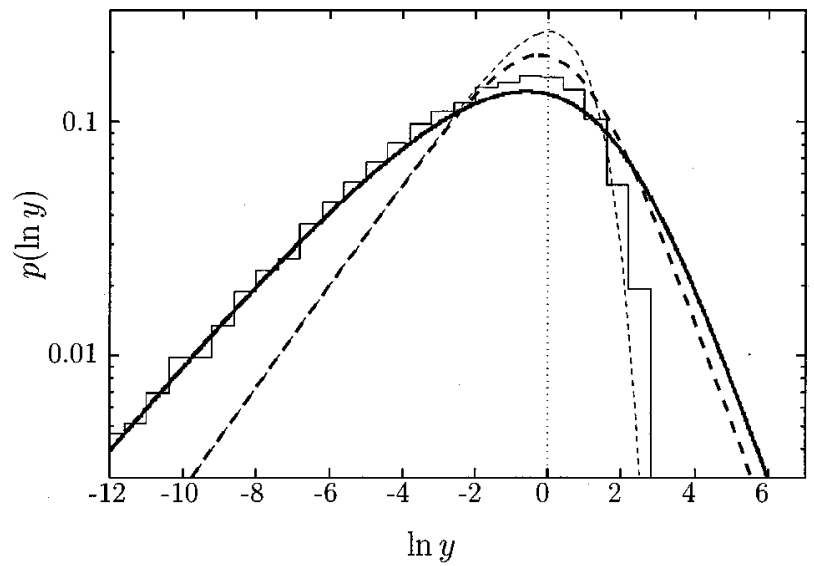

FIG. 4. Distributions of the logarithms of the widths in the strong coupling limit for the one channel case. The prediction of the two level approximation for the POE (35) (solid bold line) is compared to the numerical result (solid line) produced by diagonalizing 200 matrices at $\kappa=100$. The corresponding two level GOE result (34) (dashed bold line) is compared to the exact distribution, the Porter-Thomas curve (dashed line).

$$
p_{\mathrm{P}}(y)=\frac{1}{4 y} e^{y / \pi^{2}} W_{-1,0}\left(\frac{2 y}{\pi^{2}}\right)
$$

where

$$
W_{-1,0}(z)=\frac{2 z}{\sqrt{\pi}}\left[\left(\sqrt{z}+\frac{1}{\sqrt{z}}\right) K_{0}\left(\frac{z}{2}\right)-\sqrt{z} K_{1}\left(\frac{z}{2}\right)\right]
$$

is the Whittaker function [26], and $K_{0}(z)$ and $K_{1}(z)$ are the modified Bessel functions [27]. In Fig. 4 both distributions are compared to the Porter-Thomas distribution $\chi_{K=1}^{2}$ (28) and the numerically obtained width distribution for the POE case. Note that we plotted $p(\ln y)$ instead of $p(y)$ for all distributions in this figure, and we use a logarithmic scale on the abscissa. This is done in order to give an overall view and allowing at the same time for the recognition of the interesting features discussed in the following.

Comparing Eq. (34) to the Porter-Thomas distribution, we find that it agrees exactly in the leading power for $y \ll 1$. For large $y \gg 1 p_{\mathrm{G}}$ fails: it has a $y^{-2}$ tail which actually makes the distribution non-normalizable.

For the POE case we observe the same features when comparing Eq. (35) to the width distribution obtained numerically. For $y \rightarrow \infty, W_{-1,0}(z) \rightarrow \exp (-z / 2) / z$ leading again to a $y^{-2}$ tail being inconsistent with the numerical result. On the other hand, for $y \rightarrow 0, p_{\mathrm{P}}(y)$ fits very well to the numerical distribution. Here $K_{1}(z) \rightarrow 1 / z$ and $K_{0}(z) \rightarrow-\ln z$ and, therefore,

$$
p_{\mathrm{P}}(y) \rightarrow-\frac{\ln y}{\pi \sqrt{2 \pi y}} .
$$

In the relevant range $y \approx 1$ both distributions $p_{\mathrm{G}}(y)$ and $p_{\mathrm{P}}(y)$ show qualitatively how the level repulsion parameter $\beta$ (10) affects the width distribution of the trapped reso- 
nances. Disregarding the tails, one can clearly see from Fig. 4 the broadening in the POE case $(\beta=0)$ in comparison to the $\operatorname{GOE}(\beta=1)$.

\section{CORRELATIONS IN THE POSITIONS OF THE RESONANCES}

In order to measure the correlations between the positions of the resonances, we apply the number variance $\Sigma^{2}$ [21]. We investigate the GOE and the POE spectra in the different coupling regimes: weak, critical, and strong coupling. For the GOE similar investigations have already been done in [28].

$\Sigma^{2}$ measures the deviation of the accumulated level density from a straight line. Small $\Sigma^{2}$ is a signature for high rigidity of the level sequence. In this case the level spacings are more or less equal. On the other hand, a completely uncorrelated sequence, as, for example, the POE spectrum has minimal rigidity and therefore maximal $\Sigma^{2}$.

In contrast to [10] we focus on the few channel case (typically $K=3$ ), because we are mainly interested in the differences the GOE and the POE show at large coupling. Note that one result of [10] was that the POE spectrum at strong coupling becomes more and more GOE-like with increasing number of channels.

For the numerical calculations we implemented the following technical steps: The spectra are unfolded to constant mean level spacing $d=1$. This is done by a polynomial fit of the accumulated level density. Then the edges of the spectrum are skipped, which reduces the number of resonances by approximately $25 \%$. Furthermore, when $\kappa>1$, the $K$ broadest resonances are omitted. For the new reduced spectrum $\left\{e_{i}\right\}$ consisting now of $N^{\prime}$ levels the correlations are investigated.

For an unfolded sequence $\left\{e_{i}\right\}$, the mean number of levels found in an interval of length $l$ is $\langle n(l)\rangle=l$ due to $d=1$. The number variance $\Sigma^{2}(l)$ is defined as the variance of $n(l)$ in the ensemble mean

$$
\Sigma^{2}(l)=\left\langle n^{2}(l)\right\rangle-\langle n(l)\rangle^{2}=\left\langle n^{2}(l)\right\rangle-l^{2} .
$$

For the GOE and the POE (completely uncorrelated sequence) this quantity is known analytically [21]. Instead, for our purposes, it is sufficient to use the following approximate expressions:

For the GOE

$$
\Sigma_{\mathrm{G}}^{2}(l) \approx \frac{2 \ln l}{\pi^{2}}+0.442,
$$

and for the POE

$$
\Sigma_{\mathrm{P}}^{2}(l) \approx l
$$

In Fig. 5 we show numerical calculations of $\Sigma^{2}$ for the GOE (a) and the POE (b) spectra. This is done for three different values of the overlapping parameter $\kappa=0.1,1$, and 10. The smallest and the largest value are chosen such that a further decrease or increase of $\kappa$ does not change any more the outcome for $\Sigma^{2}$ significantly.

$\Sigma^{2}$ for the GOE [Fig. 5(a)] shows good agreement with $\Sigma_{\mathrm{G}}^{2}(39)$ for $\kappa=0.1$ and $\kappa=10$. Only in the critical region we

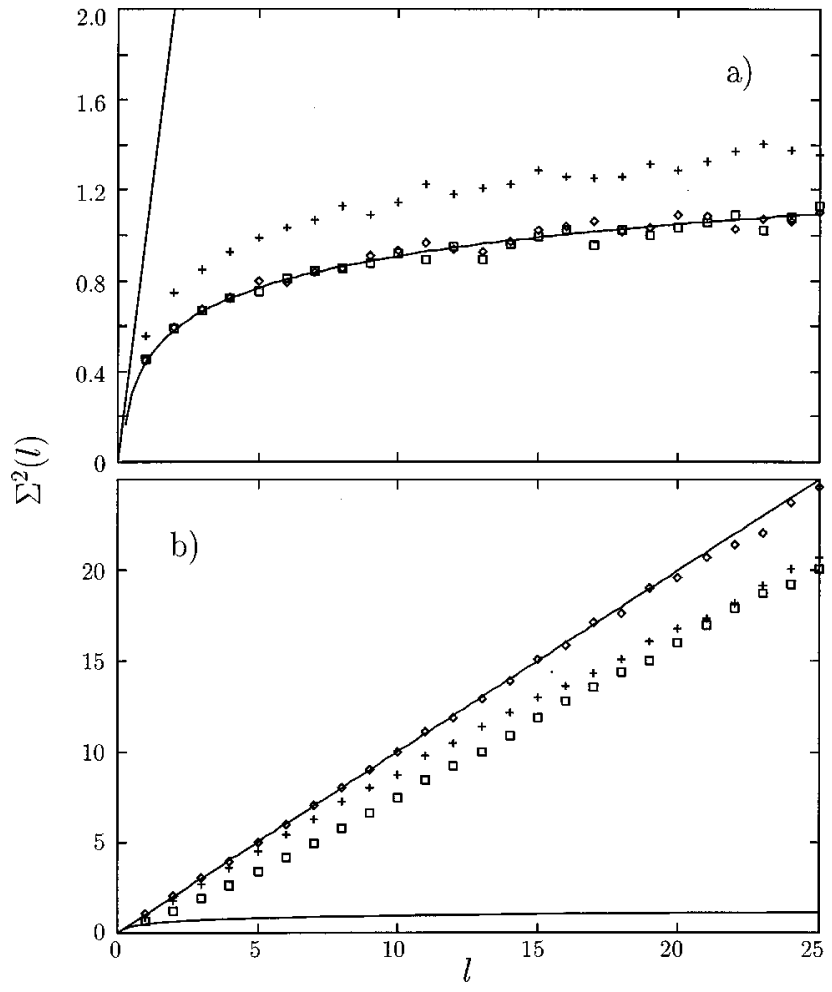

FIG. 5. Number variance $\Sigma^{2}(l)$ for three different values of the overlapping parameter $\kappa=0.1$ (diamonds), $\kappa=1.0$ (crosses), and $\kappa=10$ (squares). (a) GOE and (b) POE. The solid lines show the theoretical curves: $\Sigma_{\mathrm{G}}^{2}$ (a) and $\Sigma_{\mathrm{P}}^{2}(\mathrm{~b})$.

notice a deviation, where $\Sigma^{2}$ becomes slightly larger for all $l>1$. This decorrelation is due to the additional "degree of freedom" the resonance poles encounter in this regime [29]. In contrast to the two other cases, where the poles are restricted to a small stripe along the real axis, they now have enough space in the direction of the imaginary axis in order to avoid close neighbors. This "width repulsion" cannot be detected by observing the positions alone.

In [30] this decorrelation effect is investigated numerically for a similar system with the following result: Although the level repulsion of the poles in the complex plane increases with the number of channels, the level repulsion of the positions of the poles decreases. An explanation of the correlation measures to counteract each other is still outstanding [32].

In the POE case [Fig. 5(b)] all correlations are induced by the coupling to the decay channels. Therefore they grow with increasing $\kappa$. After completion of the redistribution (i.e., when the trapped resonances come sufficiently close to the real axis again) the $\Sigma^{2}$ curve becomes stable. This happens at $\kappa=10$. Note that the deviations from the original straight line do not vanish nor does the curve at large $\kappa$ coincide with that of the GOE. Furthermore, it is remarkable that at $\kappa<1$ the rigidity increases mainly for large $l$ and catches up at $\kappa>1$ for small $l$.

\section{CORRELATIONS CONNECTED WITH THE WIDTHS}

In [31] the number variance is generalized to an intensity variance by weighting each level with the intensity of its line as it appears in the cross section. Here we do the weighting 
with the widths of the resonances (in the one channel case both ways are identical). We denote this quantity by $\Sigma_{g}^{2}(l)$. It gives the variance of the summed width in a given interval of length $l$. Neglecting possible correlations with the widths we relate $\Sigma_{g}^{2}(l)$ to the simple number variance. Then we compare the numerical results with this formula. Occurring discrepancies indicate the existence of exactly those correlations which had been neglected in the beginning.

Following [31], we define the width-weighted stick spectrum

$$
\rho_{g}(x)=\sum y_{i} \delta\left(x-e_{i}\right), \quad \int \rho_{g}(x) d x=N^{\prime}
$$

with the normalized widths $y_{i}=\Gamma_{i} /\langle\Gamma\rangle$. For $\kappa>1$ the $K$ broad resonances are again skipped. The summed width in an interval $\Delta$ of length $l$ is

$$
B(l)=\int_{\Delta} \rho_{g}(x) d x
$$

and consequently the variance of the summed width is

$$
\Sigma_{g}^{2}(l)=\left\langle B^{2}(l)\right\rangle-\langle B(l)\rangle^{2} .
$$

Now, we relate this quantity to the number variance $\Sigma^{2}(l)$ in the following way:

$$
\begin{aligned}
B^{2}(l) & =\sum_{i j} y_{i} y_{j} \int_{\Delta^{2}} \delta\left(x-e_{i}\right) \delta\left(y-e_{j}\right) d x d y \\
& =\sum_{i} y_{i}^{2}\left\{\begin{array}{cc}
1 & E_{i} \in \Delta \\
0 & \text { otherwise }
\end{array}+\sum_{i \neq j} y_{i} y_{j} \int_{\Delta^{2}} \delta\left(x-e_{i}\right) \delta\left(y-e_{j}\right) d x d y .\right.
\end{aligned}
$$

Assuming no position-width correlations, one may perform the averages in Eq. (43) separately

$$
\langle B(l)\rangle=\langle y\rangle\langle n(l)\rangle,
$$

and, furthermore, assuming no width-width correlations, one obtains

$$
\begin{aligned}
\left\langle B^{2}(l)\right\rangle= & \left\langle y^{2}\right\rangle\langle n(l)\rangle+\langle y\rangle^{2} \\
& \times \sum_{i \neq j}\left\langle\int_{\Delta^{2}} \delta\left(x-e_{i}\right) \delta\left(y-e_{j}\right) d x d y\right\rangle .
\end{aligned}
$$

It is $\langle n(l)\rangle=l$ because the mean level distance is one. The corresponding expression without width weighting is

$$
\left\langle n^{2}(l)\right\rangle=\langle n(l)\rangle+\sum_{i \neq j}\left\langle\int_{\Delta^{2}} \delta\left(x-e_{i}\right) \delta\left(y-e_{j}\right) d x d y\right\rangle .
$$

Comparing Eqs. (46) and (47) one arrives at

$$
\left\langle B^{2}(l)\right\rangle=\left\langle y^{2}\right\rangle l+\langle y\rangle^{2}\left(\left\langle n^{2}(l)\right\rangle-l\right),
$$

and finally using Eqs. (38) and (45)

$$
\Sigma_{g}^{2}(l)=\left(\Delta y^{2}\right) l+\Sigma^{2}(l) .
$$

Here $\left(\Delta y^{2}\right)$ is the variance of the width distribution. In the case that the normalized widths $y_{i}$ are $\chi_{K}^{2}$ distributed, $\left(\Delta y^{2}\right)=2 / K$.

From Eq. (49) we see that we may clearly separate properties stemming from the correlations in the energy spectrum, as well as from the width distribution on one hand, and correlations between energies and widths or between widths at different energies on the other hand. To achieve this we proceed [31] to create from the true width-weighted spectrum (41) a synthetic one

$$
\tilde{\rho}_{g}=\sum y_{\pi(i)} \delta\left(x-\varepsilon_{i}\right)
$$

where we apply a random permutation $\pi$ to the indices of the widths. As Eq. (49) only uses the independence of widths and levels the synthetic spectrum should obey this equation, while the true one $\rho_{g}$ may not if correlations exist.

Figure 6 shows the theoretical curves $\Sigma_{\mathrm{P}}^{2}(40)$ and $\Sigma_{\mathrm{G}}^{2}(39)$ as well as $\Sigma^{2}$ for the numerically obtained energy spectrum for three channels and $\kappa=100$. We, furthermore, show $\Sigma_{g}^{2}-\left(\Delta y^{2}\right) l$ for both the true and the synthetic (decorrelated) width-weighted stick spectra under the same conditions. The result for the synthetic spectrum agrees very well with that for the energy spectrum, as it should be according to Eq. (49). The $\Sigma_{g}^{2}$ obtained for the true spectrum, on the other

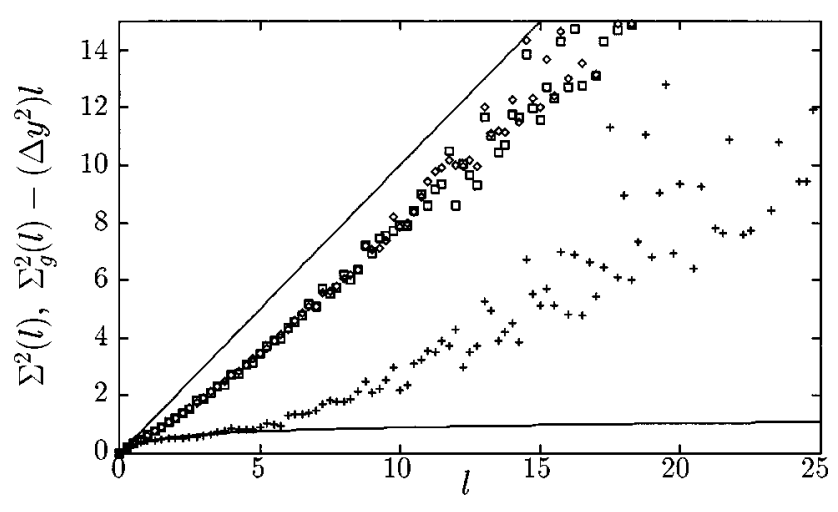

FIG. 6. Variance $\Sigma_{g}^{2}(l)$ of the summed width subtracted by $\left(\Delta y^{2}\right) l$ for the POE case $(\kappa=100)$. The crosses denote this quantity for the original spectrum which contains all correlations. The squares correspond to the synthetic spectrum and the diamonds denote the pure number variance $\Sigma^{2}$. The solid lines show the theoretical curves $\Sigma_{\mathrm{G}}^{2}$ and $\Sigma_{\mathrm{P}}^{2}$. 


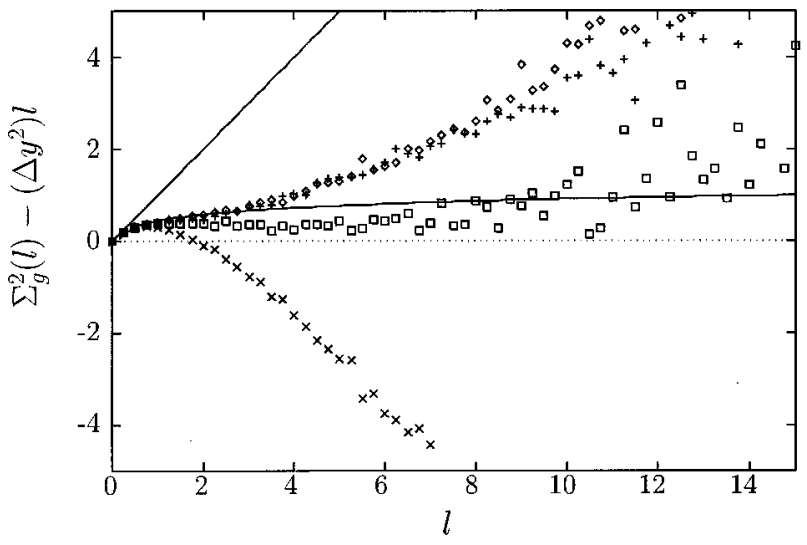

FIG. 7. Variance $\Sigma_{g}^{2}(l)$ of the summed width subtracted by $\left(\Delta y^{2}\right) l$ for the original spectrum, containing all correlations ( $\kappa=100$ ). The diamonds denote the case $K=6$, the upright crosses $K=3$, the squares $K=2$, and the crosses $K=1$. The solid lines again show $\Sigma_{\mathrm{G}}^{2}$ and $\Sigma_{\mathrm{P}}^{2}$.

hand, differs greatly. This is a clear indication that the spectrum in this case presents strong correlations, which must be contrasted to the chaotic case where a similar analysis indicates the absence of correlations. Note however that the agreement of $\Sigma_{g}^{2}-\left(\Delta y^{2}\right) l$ with $\Sigma_{\mathrm{G}}^{2}$ for $l<5$ is only accidental, what can be concluded from the results for different numbers of channels in Fig. 7.

The nature of these correlations is such that the spectrum becomes more rigid due to the width weighting [disregarding the term $\left.\left(\Delta y^{2}\right) l\right]$. This may be understood from Eq. (32) meaning that the width of a trapped resonance is more likely to be large if the distance between its neighboring levels is large, too $\Gamma_{\nu} \sim\left|\varepsilon_{\nu}-\varepsilon_{\nu-1}\right|^{2}$.

Furthermore, we expect that for increasing number $K$ of channels the effect of the position width and the width-width correlations vanishes, because the variance of the width distribution $2 / K$ becomes very small. In this case the sum of the normalized widths becomes more and more equal to the number of levels in the interval considered. This is verified in Fig. 7.

\section{SUMMARY}

We considered the distortion of a regular system by its coupling $\kappa$ to the continuum of decay channels. We obtained results for the following.

(i) The width distribution, as it is altered with increasing $\kappa$. In the weak coupling limit for both the GOE and the POE, the widths are $\chi_{K}^{2}$ distributed when normalized to unit mean. Then with increasing $\kappa$ their distributions become broader. For the GOE, the widths return to their original distribution in the strong coupling limit. For the POE, the width distribution becomes approximately a $\chi_{q}^{2}$ distribution again, but in contrast to the GOE with a much larger variance as before (cf. Fig. 2 and Fig. 3).

(ii) The correlations between the positions alone. For this analysis we used the number variance $\Sigma^{2}$. For the GOE we found correlations, as they are typical for the level statistics of a closed system in both the weak and the strong coupling region. Only in the critical region do the correlations become weaker. The POE starts without correlations. Then they in- crease steadily with the coupling parameter $\kappa$ (cf. Fig. 5).

(iii) The correlations connected with the widths. Here we used the generalized measure $\Sigma_{g}^{2}$ [31], calculated from a width-weighted stick spectra. Two additional types of correlations appear, namely, between the position and the width of each resonance and between the widths of different resonances. In the GOE case they appear only in the critical region, whereas in the $\mathrm{POE}$ case they increase steadily (cf. Figs. 6 and 7).

Furthermore, we derived an analytic expression for the width distribution of the GOE and POE in the one channel case at strong coupling (cf. Fig. 4). This is achieved by relating the width of a trapped state to the distance of the two neighbored levels. In this way, the different width distribution of the GOE and POE is explained.

The results of our investigations show the special role of the GOE. Its properties survive the distortion of the system by coupling it to the continuum: at large coupling the correlations and the width distribution are the same as at low coupling. In contrast to the GOE, the properties of the POE are not restored at the strong coupling strength.

Realistic systems are often in the critical region where correlations in the spectrum are induced by the coupling to the continuum. Under these conditions the $S$-matrix poles are difficult to find. Nevertheless, they determine the statistical properties of the cross section. We will investigate this problem in a forthcoming paper for both the GOE and the POE.

\section{ACKNOWLEDGMENT}

Valuable discussions with G. Soff and V. V. Sokolov are gratefully acknowledged. The investigations are supported by DFG (Ro 922/6) and by DAAD.

\section{APPENDIX A: RESONANCE WIDTH IN THE TWO LEVEL APPROXIMATION}

Writing Eq. (31) for two neighboring levels, we get

$$
\frac{v_{1}^{2}}{s+\frac{\Delta}{2}}+\frac{v_{2}^{2}}{s-\frac{\Delta}{2}}=0, \quad \frac{v_{1}^{2}}{\left(s+\frac{\Delta}{2}\right)^{2}}+\frac{v_{2}^{2}}{\left(s-\frac{\Delta}{2}\right)^{2}}=\frac{4}{\eta \Gamma},
$$

where $\Delta=\varepsilon_{2}-\varepsilon_{1}$ is the distance between the levels and $E$ is substituted by $E=\frac{1}{2}\left(\varepsilon_{1}+\varepsilon_{2}\right)+s$. Due to the first equation

$$
s=-\frac{\Delta}{2} \frac{v_{2}^{2}-v_{1}^{2}}{v_{1}^{2}+v_{2}^{2}}=-\frac{\Delta}{2} \tau .
$$

Inserting this result into the second equation of (A1) yields

$$
\frac{\Delta^{2}}{\eta \Gamma}=\frac{v_{1}^{2}}{(1-\tau)^{2}}+\frac{v_{2}^{2}}{(1+\tau)^{2}}=\frac{v_{1}^{2}+v_{2}^{2}}{1-\tau^{2}}
$$

Finally, measuring $\Delta$ in units of the mean level spacing $\Delta=d_{0} s$ in the center of the spectrum, and $\Gamma$ in units of the mean width $\Gamma=\left\langle\Gamma_{\nu}\right\rangle y$ according to Eq. (23), one arrives at Eq. (32) 


$$
y=\frac{\pi^{2} s^{2}}{4 N\left(v_{1}^{2}+v_{2}^{2}\right)}\left(1-\tau^{2}\right) .
$$

\section{APPENDIX B: WIDTH DISTRIBUTION}

The width distribution according to Eq. (32) is

$$
\begin{gathered}
p(y)=\frac{N}{2 \pi} \int d v_{1} d v_{2} e^{-N / 2\left(v_{1}^{2}+v_{2}^{2}\right)} \int d s P(s) \\
\times \delta\left[y-\frac{\pi^{2} s^{2}}{4 N\left(v_{1}^{2}+v_{2}^{2}\right)}\left(1-\tau^{2}\right)\right] .
\end{gathered}
$$

Transforming $v_{1}$ and $v_{2}$ into spherical coordinates $r / \sqrt{N} \cos \phi$ and $r / \sqrt{N} \sin \phi$ so that $\tau=\sin ^{2} \phi-\cos ^{2} \phi$ $=-\cos 2 \phi$, we get

$$
\begin{gathered}
p(y)=\frac{1}{2 \pi} \int r d r d \phi e^{-r^{2} / 2} \int d s P(s) \\
\quad \times \delta\left[y-\frac{\pi^{2} s^{2} \sin ^{2} 2 \phi}{4 r^{2}}\right] .
\end{gathered}
$$

It is enough to integrate $\phi$ from 0 to $\pi / 4$ because of the eightfold symmetry of the integrand. Applying, in addition, the transformation $x=\sin ^{2} 2 \phi$

$$
\begin{aligned}
p(y)= & \frac{1}{\pi} \int r d r e^{-r^{2} / 2} \int_{0}^{1} \frac{d x}{\sqrt{x(1-x)}} \int d s P(s) \\
& \times \delta\left[y-\frac{\pi^{2} s^{2} x}{4 r^{2}}\right] .
\end{aligned}
$$

In order to go ahead we consider the two cases GOE and POE separately in the following subsections.

\section{GOE case}

Here the nearest neighbor distribution reads

$$
P_{\mathrm{G}}(s)=\frac{\pi}{2} s e^{-\pi s^{2} / 4}
$$

In order to resolve the $\delta$ function, we substitute $r$ as a function of $y^{\prime}=\pi^{2} s^{2} x /\left(4 r^{2}\right)$ and use $\int \delta\left(y-y^{\prime}\right) f\left(y^{\prime}\right) d y=f(y)$. This leads to

$$
p_{\mathrm{G}}(y)=\frac{\pi^{2}}{16 y^{2}} \int_{0}^{1} d x\left(\frac{x}{1-x}\right)^{1 / 2} \int_{0}^{\infty} d s s^{3} e^{-\alpha s^{2}}
$$

$$
\alpha=\frac{\pi}{4}\left(1+\frac{\pi x}{2 y}\right)
$$

The integral over the level spacing $s$ gives $1 /\left(2 \alpha^{2}\right)$ and it remains a last integration, namely,

$$
p_{\mathrm{G}}(y)=\frac{2}{\pi^{2}} \int_{0}^{1} d x\left(\frac{x}{1-x}\right)^{1 / 2}\left(\frac{2 y}{\pi}+x\right)^{-2} .
$$

This may be solved by substituting $t=\sqrt{x / 1-x}$

$$
p_{\mathrm{G}}(y)=\frac{4}{\pi^{2}(1+b)^{2}} \int_{0}^{\infty} d t \frac{t^{2}}{\left(t^{2}+\frac{b}{b+1}\right)^{2}}, \quad b=\frac{2 y}{\pi}
$$

and integrating by parts. Then

$$
p_{\mathrm{G}}(y)=\frac{1}{\pi(1+b)^{2}}\left(\frac{1+b}{b}\right)^{1 / 2}=\frac{1}{\sqrt{2 \pi y}}\left(1+\frac{2 y}{\pi}\right)^{-3 / 2} \text {. }
$$

\section{POE case}

Here the nearest neighbor distribution is

$$
P_{\mathrm{P}}(s)=e^{-s} \text {. }
$$

In contrast to the GOE case we first substitute $s$ instead of $r$. It follows that

$$
\begin{aligned}
p_{\mathrm{P}}(y) & =\frac{1}{\pi^{2} \sqrt{y}} \int r^{2} d r e^{-r^{2} / 2} \int_{0}^{1} \frac{d x}{x \sqrt{1-x}} e^{-2 r / \pi \sqrt{y / x}} \\
& =\frac{2}{\pi^{2} \sqrt{y}} \int d r r^{2} e^{-r^{2} / 2} \int_{1}^{\infty} \frac{d z e^{-\alpha z}}{\sqrt{z^{2}-1}}, \quad \alpha=\frac{2 r}{\pi} \sqrt{y} .
\end{aligned}
$$

The last integral represents the modified Bessel function [27]

$$
p_{\mathrm{P}}(y)=\frac{2}{\pi^{2} \sqrt{y}} \int d r r^{2} e^{-r^{2} / 2} K_{0}\left(\frac{2 \sqrt{y}}{\pi} r\right)
$$

This integral can be found in [26]

$$
p_{\mathrm{P}}(y)=\frac{1}{4 y} e^{\left(y / \pi^{2}\right)} W_{-1,0}\left(2 y / \pi^{2}\right) \text {. }
$$

[1] H. Feshbach, Ann. Phys. (N.Y.) 5, 357 (1958).

[2] H. Feshbach, Ann. Phys. (N.Y.) 19, 287 (1962).

[3] C. Mahaux and H. A. Weidenmüller, Shell-Model Approach to Nuclear Reactions (North-Holland, Amsterdam, 1969).

[4] P. Kleinwächter and I. Rotter, Phys. Rev. C 32, 1742 (1985).
[5] V. V. Sokolov and V. G. Zelevinsky, Nucl. Phys. A 504, 562 (1989).

[6] F. Haake, F. Izrailev, N. Lehmann, D. Saher, and H.-J. Sommers, Z. Phys. B 88, 359 (1992).

[7] T. A. Brody, J. Flores, J. B. French, P. A. Mello, A. Pandey, 
and S. S. M. Wong, Rev. Mod. Phys. 53, 385 (1981).

[8] Statistical Theories of Spectra: Fluctuations, edited by C. E. Porter (Academic, New York, 1965).

[9] A. Müller and H. L. Harney, Phys. Rev. C 35, 1228 (1987).

[10] F.-M. Dittes, I. Rotter, and T. H. Seligman, Phys. Lett. A 158, 14 (1991).

[11] S. Albeverio, F. Haake, P. Kurasov, M. Kuś, and P. Šeba, J. Math. Phys. (N.Y.) 37, 4888 (1996).

[12] H.-J. Stöckmann and J. Stein, Phys. Rev. Lett. 64, 2215 (1990).

[13] F. Haake, G. Lenz, P. Šeba, J. Stein, H.-J. Stöckmann, and K. Życzkowski, Phys. Rev. A 44, R6161 (1991).

[14] C. M. Marcus, R. M. Westervelt, P. F. Hopkins, and A. C. Gossard, CHAOS 3, 643 (1993).

[15] C. Jung and T. H. Seligman, Phys. Rep. 285, 80 (1997).

[16] Y. Alhassid and R. D. Levine, Phys. Rev. Lett. 57, 2879 (1986).

[17] Y. Alhassid and M. Feingold, Phys. Rev. A 39, 374 (1989).

[18] F.-M. Dittes, H. L. Harney, and A. Müller, Phys. Rev. A 45, 701 (1992).

[19] E. Persson, T. Gorin, and I. Rotter, Phys. Rev. E 54, 3339 (1996).

[20] Y. V. Fyodorov and H.-J. Sommers, Pis'ma Zh. Eskp. Teor. Fiz. 63, 970 (1996) [ JETP Lett. 63, 970 (1996)].
[21] O. Bohigas, in Chaos and Quantum Physics, edited by M. J. Giannoni Proceedings of the Les Houches Summer School, Session LII (North-Holland, Amsterdam, 1991), p. 91.

[22] I. Rotter, Rep. Prog. Phys. 54, 635 (1991).

[23] V. V. Sokolov and V. G. Zelevinsky, Ann. Phys. (N.Y.) 216, 323 (1992).

[24] F. Lorenz, Lineare Algebra II (BI-Wissenschaftsverlag, Mannheim, 1989).

[25] J. J. M. Verbaarschot, H. A. Weidenmüller, and M. R. Zirnbauer, Phys. Rep. 129, 367 (1985).

[26] I. S. Gradshteyn and I. M. Ryzhik, Table of Integrals, Series and Products (Academic, San Diego, 1980).

[27] Handbook of Mathematical Functions, edited by M. Abramowitz and I. A. Stegun (Dover, New York, 1970).

[28] S. Mizutori and V. G. Zelevinsky, Z. Phys. A 346, 1 (1993).

[29] M. Müller, F.-M. Dittes, W. Iskra, and I. Rotter, Phys. Rev. E 52, 5961 (1995).

[30] S. Drożdż, A. Trellakis, and J. Wambach, Phys. Rev. Lett. 76, 4891 (1996).

[31] M. Lombardi and T. H. Seligman, Phys. Rev. A 47, 3571 (1993).

[32] A paper providing analytical results, which may be relevant in this context, appeared just recently: Fyodorov et al. cond-mat/9703152. 